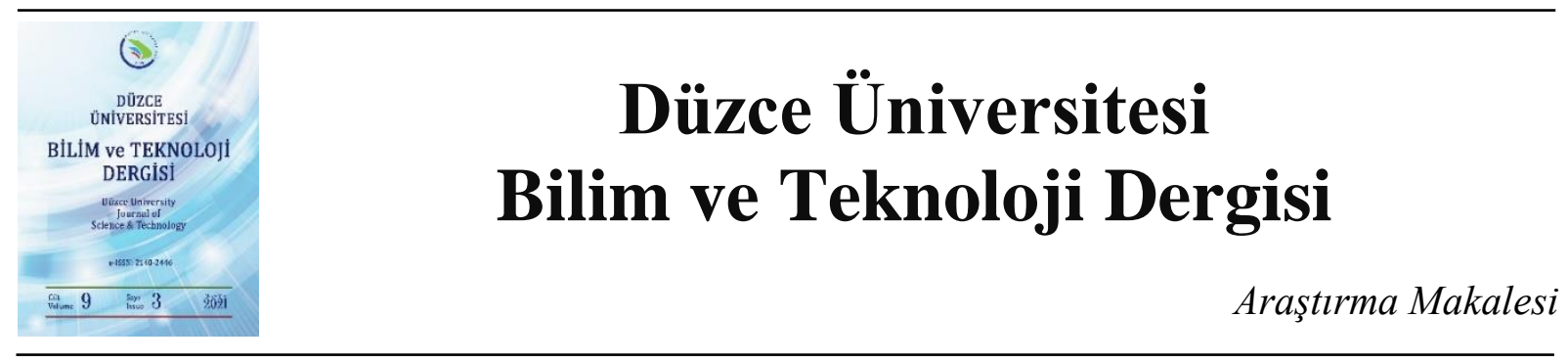

\title{
Bazı Karayosunu Türlerinin In Vitro Antimikrobiyal Aktivitelerinin Belirlenmesi
}

\author{
Atakan BENEK a,*, (D) Merve ŞENTURAN ${ }^{\text {, }}$ (iD) Özcan ŞiMŞEK ${ }^{\mathrm{c}}$, (D) Kerem CANLI ${ }^{\mathrm{d}}$, \\ Ergin Murat ALTUNER ${ }^{\mathrm{e}}$ \\ ${ }^{a}$ Biyoloji Bölümü, Fen Bilimleri Enstitüsü, Kastamonu Üniversitesi, Kastamonu, TÜRKİYE \\ ${ }^{b}$ Biyoloji Bölümü, Fen Bilimleri Enstitüsü, Kastamonu Üniversitesi, Kastamonu, TÜRKIYE \\ ${ }^{c}$ Ormancllkk Bölümü, Yenice Meslek Yüksekokulu, Çanakkale 18 Mart Üniversitesi, Çanakkale, TÜRKIYE \\ ${ }^{d}$ Biyoloji Bölümü, Fen Fakültesi, Dokuz Eylül Üniversitesi, İzmir, TÜRKIYE \\ ${ }^{e}$ Biyoloji Bölümü, Fen Fakültesi, Kastamonu Üniversitesi, Kastamonu, TÜRKIYY \\ * Sorumlu yazarin e-posta adresi: atakan.benek@hotmail.com
}

DOI: 10.29130/dubited.872689

\begin{abstract}
ÖZET
Karayosunlarının hastalıklara karşı etkisi olduğu çok uzun yıllardan beri bilinmektedir ve yaraların tedavi edilmesi ve enfeksiyonlar ile mücadele gibi alanlarda etkin olarak kullanılmaktadırlar. Bu çalışmada Plagiomnium elatum, Rhynchostegium megapolitanum, Tortella fasciculata ve Tortella tortuosa karayosunu örneklerinin etil alkol ekstraktları disk difüzyon yöntemi kullanılarak, 8 gram pozitif, 9 gram negatif bakteri ve 1 maya olmak üzere, toplam 18 mikroorganizma üzerinde test edilerek antimikrobiyal aktiviteleri belirlenmiştir. Disk difüzyon testi sonucunda tüm karayosunu örneklerinin birkaç mikroorganizmaya karşı antimikrobiyal aktiviteye sahip olduğu ve özellikle gram pozitif olan Staphylococcus aureus ATCC 25923 ve gram negatif olan Escherichia coli ATCC 25922 üzerinde belirgin bir antimikrobiyal aktivitesinin olduğu ortaya konmuştur.
\end{abstract}

Anahtar Kelimeler: Plagiomnium elatum, Rhynchostegium megapolitanum, Tortella fasciculata, Tortella tortuosa, Antimikrobiyal aktivite, Disk difüzyon

\section{Determination Of In Vitro Antimicrobial Activities of Some Moss Species}

\section{ABSTRACT}

Mosses have been known for many years to be effective against diseases and they are successfully used in areas such as treating wounds and fighting infections. In this study, ethyl alcohol extracts of Plagiomnium elatum, Rhynchostegium megapolitanum, Tortella fasciculata and Tortella tortuosa were tested using disk diffusion method on a total of 18 microorganisms, 8 gram positive, 9 gram negative bacteria and 1 yeast, and their antimicrobial activities were determined. As a result of the disk diffusion test, it was demonstrated that all moss samples had antimicrobial activity against several strains, but they had significant antimicrobial activities especially on gram positive Staphylococcus aureus ATCC 25923 and gram negative Escherichia coli ATCC 25922.

Keywords: Plagiomnium elatum, Rhynchostegium megapolitanum, Tortella fasciculata, Tortella tortuosa, Antimicrobial activity, Disk diffusion 


\section{GIRIS}

Günümüzde artan Dünya nüfusu, gıda ürünlerinde azalan doğallık ve endüstriyel atıkların giderek artması sonucunda sağlık problemleri de hızla artmaktadır. Toplumların en büyük sağlık problemlerinden biri de enfeksiyon hastalıklarıdır. Enfeksiyon hastalıkları ile mücadelede kullanılan doğal veya sentetik olarak üretilen antibiyotiklerin, mikroorganizmaların direnç oluşturmaları nedeniyle etkisiz kalmaya başlamasının yanı sıra, yan etkilerinin ortaya konması bilim insanlarının yeni antimikrobiyal etkiye sahip maddeleri keşfetmesi ve yeni sağlık stratejileri geliştirmesini zorunlu hale getirmiştir [1,2].

Tarih boyunca hastalıkların tedavisinde ve enfeksiyonlar ile mücadelede bitkiler kullanılmıştır. Dünya Sağlık Örgütü (WHO) tarafından dünya genelinde 20.000 bitkinin tedavi amaçlı kullanıldığı rapor edilmiştir [3]. Tedavi amacıyla kullanılan bitki gruplarından biri de Musci (Karayosunları), Hepaticae (Tallussu ve yapraksı ciğerotları), Anthocerotae (Boynuzsu ciğerotları) sınıflarını içinde bulunduran yaklaşı 1.036 cins ve 18.409 türden oluşan Briyofitlerdir [4]. Briyofitler içerisinde yer alan karayosunlarının çok eski dönemlerden beri iyileştirici özelliği bilinmekte ve tedavi amacıyla kullanılmaktadır [5].

Karayosunlarının Dünya genelinde yaklaşık 12.700 türü ülkemizde ise 7 türü endemik olmak üzere toplam 835 türü bulunmaktadır [6,7]. Ekosistemin önemli parçalarından biri olan karayosunları, genellikle ekonomik değeri fazla olmayan ve büyük bir kısmının ayırt edilebilmesi zor olan küçük tohumsuz bitkilerdir [8]. Karayosunları üzerine yapılan çalışmalar, basit yapılarına rağmen karayosunlarının sahip oldukları sekonder metabolitlerin bazılarının yaraların iyileşmesinde etkili olduğu ve bazı mikroorganizmalara karş1 antimikrobiyal etki gösterdiklerini ortaya koymuştur [9]. $\mathrm{Bu}$ çalışmada Plagiomnium elatum (Bruch \& Schimp.) T.J.Kop., Rhynchostegium megapolitanum (Blandow. ex F. Weber \& D. Mohr) Schimp, Tortella fasciculata (Culm.) Culm. ve Tortella tortuosa (Hedw.) Limpr karayosunu örneklerinin etanol ekstraktlarının 18 mikroorganizma üzerinde antimikrobiyal aktiviteleri belirlenmiştir.

\section{MATERYAL METOD}

\section{A. KARAYOSUNU ÖRNEKLERİ}

$\mathrm{Bu}$ çalışmada kullanılan karayosunu örnekleri Doçent Doktor Kerem Canlı tarafından Amasya Akdağ'dan toplanmış ve tür teşhisleri yapılmıştır. Şahit örnekler Dokuz Eylül Üniversitesi, Biyoloji Bölümü’nde saklanmıştır.

Tablo 1. Bu çalışmada kullanılan bryofitlerin lokasyonları.

\begin{tabular}{|c|c|c|c|c|}
\hline & Karayosunu Türleri & Lokasyon & Rakım & Koordinat \\
\hline 1 & Plagiomnium elatum & Akdağ, Amasya & 872 & $\begin{array}{l}\mathrm{N} 40^{\circ} 41.006^{\prime} \text { E } 036^{\circ} \\
3,902^{\prime}\end{array}$ \\
\hline 2 & $\begin{array}{l}\text { Rhynchostegium } \\
\text { megapolitanum }\end{array}$ & Akdağ, Amasya & 498 & $\begin{array}{l}\mathrm{N} 40^{\circ} 41.154^{\prime} \text { E } 035^{\circ} \\
57,471^{\prime}\end{array}$ \\
\hline 3 & Tortella fasciculata & Akdağ, Amasya & 1550 & $\begin{array}{l}\mathrm{N} 40^{\circ} 47.935^{\prime} \text { E } 036^{\circ} \\
6,600^{\prime}\end{array}$ \\
\hline 4 & Tortella tortuosa & Akdağ, Amasya & 1650 & $\begin{array}{l}\mathrm{N} 40^{\circ} \quad 48.358^{\prime} \mathrm{E} \quad 036^{\circ} \\
5,140^{\prime}\end{array}$ \\
\hline
\end{tabular}




\section{B. EKSTRAKSIYYON YÖNTEMI}

Karayosunu örnekleri toplanıp kurutulduktan sonra 100 gram örnek blender ile toz haline alana kadar ögütülmüştür. Ögütme işleminin ardından toz haline gelen örnekler $200 \mathrm{~mL}$ saf etil alkol (SigmaAldrich) içinde 48 saat boyunca 140 ppm'de ve oda koşullarında çalkalanmıştır. 48 saatin sonunda Whatman No. 1 filtre kağıdı kullanılarak cam balonlar içerisine süzülmüştür. Süzme işleminin ardından etil alkol $30^{\circ} \mathrm{C}^{\prime}$ de döner buharlaştırıcı (Buchi R3) ile vakum altında buharlaştırılmıştır. Buharlaştırma işleminin tamamlanmasından sonra ölçümler yapılmış, kalıntılar toplanmış ve ekstrakt hazırlamak için kullanılmıştır [10]. P. elatum $180 \mathrm{mg}, 15 \mathrm{~mL}$ etanol içerisinde, $R$. megapolitanum $170 \mathrm{mg}, 15 \mathrm{~mL}$ etanol içerisinde, T. fasciculata $50 \mathrm{mg}, 5 \mathrm{~mL}$ etanol içerisinde ve $T$. tortuosa $50 \mathrm{mg}$, etanol $10 \mathrm{~mL}$ içerisinde çözdürülerek ekstrakt stokları hazırlanmıştır.

\section{MIKROORGANIZMALAR}

P. elatum, R. megapolitanum, T. fasciculata, T. tortuosa türlerinin antimikrobiyal aktivitelerinin belirlenmesi için 8 gram pozitif, 9 gram negatif bakteri ve 1 maya toplam 18 mikroorganizma Mueller Hinton Agar (BD Difco, ABD) üzerinde zenginleştirilerek kullanılmıştır [10]. Kullanılan suşların 11'i standart bakteri, 6'sı standart dişı gıda izole bakteri ve 1 tanesi de standart maya suşudur. Beş tanesi standart gram pozitif bakterilerdir ve bunlar Bacillus subtilis DSMZ 1971, Enterecoccus faecalis ATCC 29212, Listeria monocytogenes ATCC 7644, Staphylococcus aureus ATCC 25923 ve Staphylococcus epidermidis DSMZ 20044 bakterileridir. Kalan bakterilerin altı tanesi standart gram negatif bakteridir ve Pseudomonas fluorescens P1, Escherichia coli ATCC 25922, Pseudomonas aeruginosa DSMZ 50071, Enterobacter aerogenes ATCC 13048, Salmonella enteritidis ATCC 13075, Salmonella typhimurium SL1344, türleridir. Çalışmada Candida albicans DSMZ 1386 olmak üzere 1 standart maya kullanılmıştır. Ayrıca gıdalardan izole edilen altı bakteri de çalışılmıştır. Bu bakterilerden Enterococcus faecium, Enterococcus durans, Listeria innocua gram pozitif, Klebsiella pneumoniae, Salmonella infantis ve Salmonella kentucky gram negatifdir.

\section{D. İNOKULUM HAZIRLAMA}

Antimikrobiyal aktivitesi belirlenen bakteri türleri $37^{\circ} \mathrm{C}^{\prime} \mathrm{de} 24$ saat, kullanılan tek maya türü C. albicans $27^{\circ} \mathrm{C}^{\prime}$ de 48 saat inkübe edilmiştir [11]. Bakteri türlerinde yaklaşık $10^{8} \mathrm{cfu}^{\mathrm{mL}} \mathrm{m}^{-1}$ ve C. albicans' da yaklaş1 $10^{7} \mathrm{cfu} . \mathrm{mL}^{-1}$ içeren inokülasyonları standart hale getirmek amacıyla tüm bakteri ile maya türleri steril \% 0,9 $\mathrm{NaCl}$ çözeltisi içerisinde $0,5 \mathrm{Mc}$ Farland standardına getirildikten sonra çalışma yapılmıştır $[12,13]$.

\section{E. ANTIMIKROBIYYL AKTIVITE TESTi}

Karayosunu örneklerinin etanol ekstraktlarının antimikrobiyal aktiviteleri Andrews [14] tarafindan açıklanan şekilde disk difüzyon testi ile belirlenmiştir. Başlangıç aşamasında Mueller Hinton Agar (BD Difco, ABD) 4,0 $\mathrm{mm} \pm 0,5 \mathrm{~mm}$ 'lik bir kalınlıkta olacak şekilde $90 \mathrm{~mm}$ steril petri kabına dökülmüştür. Ardından P. elatum 30, 60, $100 \mu \mathrm{L}, R$. megapolitanum 30, 60, $100 \mu \mathrm{L}, T$. fasciculata $20,30,60 \mu \mathrm{L}$ ve $T$. tortuosa 30, 60, 150 $\mu \mathrm{L}$ miktarlarında $6 \mathrm{~mm}$ yarıçapa sahip Oxoid Antimikrobiyal Duyarlılık Test Disklerine yüklenmiştir. Diskler üzerinde bulunan ve test sonuçlarına etki edebilme ihtimali olan kalan etil alkolü disklerden uçurmak için bir gece $30^{\circ} \mathrm{C}$ de steril koşullar altında kurumaya bırakılmıştır. Kuruma işleminin ardından steril $\mathrm{NaCl}$ çözeltisi içerisinde standardize edilmiş mikroorganizmalar Petri kaplarına dökülmüş olan besi yerlerinin yüzeyini tamamen kaplayacak şekilde ekimleri yapılmıştır [15]. Ekim işleminin tamamlanmasının ardından son olarak ekstrakt yüklü olan diskler mikroorganizma ekimi yapılmış olan besi yerlerinin üzerine yerleştirilerek inkübe edilmiştir. İnkübasyon süresi 
tamamlandıktan sonra disklerin etrafında oluşan inhibisyon zonlarının çapları mm olarak ölçülmü̈s kaydedilmiştir [16].

\section{F. KONTROLLER}

Ekstraksiyon solventi (etanol) ile steril boş diskler negatif kontrol olarak kullanılmıştır. Ayrıca Gentamisin antibiyotiği de pozitif kontrol olarak kullanılarak sonuçların karşılaştırılmasında kullanılmıştır.

\section{G. İSTATISTIK}

Yapılan bütün testler üç tekrarlı olacak şekilde uygulanmıştır. Deneysel çalışmalardan elde edilen veriler, tekrarlar arasındaki farkın önemini göstermek için ANOVA testi ile analiz edilmiş ve ekstraktın antimikrobiyal aktivitesi ile artan konsantrasyonlar arasında bir korelasyon olup olmadığını göstermek için Pearson korelasyon katsayısı kullanılmıştır. İstatistiksel analiz için R Studio, versiyon 3.3.2 kullanılmıştır [17].

\section{BULGULAR}

P. elatum, R. megapolitanum, T. fasciculata ve T. tortuosa türlerinin antimikrobiyal aktivitelerinin belirlenmesi amaciyla 18 mikroorganizma üzerinde yapılan disk difüzyon testinde 16 mikroorganizmaya karşı etki tespit edilmiştir. P. elatum türü S. aureus $(10 \mathrm{~mm})$, L. monocytogenes $(9$ $\mathrm{mm})$, S. enteritidis $(9 \mathrm{~mm})$, E. faecium $(8 \mathrm{~mm})$, E. coli $(8 \mathrm{~mm})$, K. pneumoniae $(8 \mathrm{~mm})$, S. epidermidis $(8 \mathrm{~mm}), P$. aeruginosa $(7 \mathrm{~mm})$ ve $E$. aerogenes $(7 \mathrm{~mm})$ suşlarına karş1 etki göstermiştir. $R$. megapolitanum türü E. coli $(9 \mathrm{~mm})$, E. aerogenes $(8 \mathrm{~mm})$, S. enteritidis $(8 \mathrm{~mm})$, S. epidermidis $(8 \mathrm{~mm})$, L. monocytogenes $(8 \mathrm{~mm})$, K. pneumoniae $(7 \mathrm{~mm})$, P. aeruginosa $(7 \mathrm{~mm})$ ve S. typhimurium $(7 \mathrm{~mm})$ suşları üzerinde etkili olmuştur. T. bambergeri türü E.aerogenes $(8 \mathrm{~mm}), S$. kentucky $(8 \mathrm{~mm})$, E. coli $(7$ $\mathrm{mm})$, P. aeruginosa $(7 \mathrm{~mm})$ suşlarına karşı etkili olmuştur. T. tortuosa türü $S$. aureus $(9 \mathrm{~mm})$, B. subtilis (9 mm), E. faecium $(8 \mathrm{~mm})$, K. pneumoniae $(8 \mathrm{~mm})$, S. epidermidis $(8 \mathrm{~mm})$, E.aerogenes $(7 \mathrm{~mm})$, E. faecalis $(7 \mathrm{~mm})$, E. coli $(7 \mathrm{~mm})$, L. innocua $(7 \mathrm{~mm})$, P. fluorescens $(7 \mathrm{~mm})$, S. enteritidis $(7 \mathrm{~mm}), S$. infantis $(7 \mathrm{~mm})$ ve $S$. kentucky $(7 \mathrm{~mm})$ suşlarına karşı etkili olduğu belirlenmiştir. Bu çalışmada pozitif kontrol olarak gentamisin antibiyotik diskleri kullanılmıştır. 
Tablo 2. Bu çalışmada kullanılan bryofitlerin disk difüzyon testi sonuçları.

\begin{tabular}{|c|c|c|c|c|c|c|c|c|c|c|c|c|c|}
\hline & \multicolumn{3}{|c|}{ Plagiomnium elatum } & \multicolumn{3}{|c|}{ Rhynchostegium megapolitanum } & \multicolumn{3}{|c|}{ Tortella fasciculata } & \multicolumn{3}{|c|}{ Tortella tortuosa } & \multirow{2}{*}{ Gentamicir } \\
\hline & $30 \mu \mathrm{L}$ & $60 \mu \mathrm{L}$ & $100 \mu \mathrm{L}$ & $30 \mu \mathrm{L}$ & $60 \mu \mathrm{L}$ & $100 \mu \mathrm{L}$ & $20 \mu \mathrm{L}$ & $30 \mu \mathrm{L}$ & $60 \mu \mathrm{L}$ & $30 \mu \mathrm{L}$ & $60 \mu \mathrm{L}$ & $150 \mu \mathrm{L}$ & \\
\hline Bacillus subtilis DSMZ 1971 & \multirow[t]{2}{*}{ - } & - & - & \multirow[t]{2}{*}{-} & - & - & - & - & - & - & \multirow{2}{*}{$7.00 \pm 0.00$} & $8.00 \pm 0.58$ & 30 \\
\hline Candida albicans DSMZ 1386 & & - & - & & - & - & - & - & - & - & & - & - \\
\hline Enterobacter aerogenes ATCC 13048 & - & - & $7.00 \pm 0.00$ & - & $8.00 \pm 0.00$ & $8.00 \pm 0.58$ & $7.00 \pm 0.00$ & $7.00 \pm 0.58$ & $8.00 \pm 0.58$ & $7.00 \pm 0.00$ & $7.00 \pm 0.00$ & $7.00 \pm 0.58$ & 23 \\
\hline Enterococcus durans & \multirow[t]{2}{*}{ - } & \multirow[t]{2}{*}{-} & - & - & - & - & - & - & - & - & - & - & 14 \\
\hline Enterococcus faecalis ATCC 29212 & & & - & - & - & - & - & - & - & - & - & $7.00 \pm 0.00$ & 13 \\
\hline Enterococcus.faecium & - & $7.00 \pm 0.00$ & $8.00 \pm 0.58$ & - & - & - & - & - & - & - & $7.00 \pm 0.00$ & $8.00 \pm 0.58$ & 28 \\
\hline Esherichia coli ATCC 25922 & $8.00 \pm 0.58$ & $7.00 \pm 0.00$ & $8.00 \pm 0.00$ & - & $7.00 \pm 0.00$ & $9.00 \pm 0.58$ & - & - & $7.00 \pm 0.00$ & - & - & $7.00 \pm 0.00$ & 20 \\
\hline Klebsiella pneumonia & $7.00 \pm 0.00$ & $8.00 \pm 0.00$ & $8.00 \pm 0.58$ & $7.00 \pm 0.00$ & $7.00 \pm 0.00$ & $7.00 \pm 0.00$ & - & - & - & $7.00 \pm 0.00$ & $7.00 \pm 0.58$ & $8.00 \pm 0.58$ & 22 \\
\hline Listeria innocua & - & - & - & - & - & - & - & - & - & - & - & $7.00 \pm 0.00$ & 23 \\
\hline Listeria monocytogenes ATCC 7644 & $7.00 \pm 0.00$ & $8.00 \pm 0.00$ & $9.00 \pm 0.00$ & $8.00 \pm 0.58$ & $7.00 \pm 0.00$ & $7.00 \pm 0.00$ & - & - & - & - & - & - & 28 \\
\hline Pseudomonas aeruginosa DSMZ 50071 & $7.00 \pm 0.00$ & $7.00 \pm 0.00$ & $7.00 \pm 0.00$ & $7.00 \pm 0.00$ & $7.00 \pm 0.00$ & $7.00 \pm 0.00$ & - & $7.00 \pm 0.00$ & $7.00 \pm 0.58$ & - & - & - & 15 \\
\hline Pseudomonas fluorescens $\mathrm{P} 1$ & - & - & - & - & - & - & - & - & - & - & - & $7.00 \pm 0.00$ & 12 \\
\hline Salmonella enteritidis ATCC 13075 & $7.00 \pm 0.00$ & $8.00 \pm 0.58$ & $9.00 \pm 0.00$ & $7.00 \pm 0.00$ & $7.00 \pm 0.00$ & $8.00 \pm 0.00$ & - & - & - & - & - & $7.00 \pm 0.00$ & 24 \\
\hline Salmonella infantis & - & - & - & - & - & - & - & - & - & $7.00 \pm 0.00$ & $7.00 \pm 0.00$ & $7.00 \pm 0.00$ & 24 \\
\hline Salmonella kentucky & - & - & - & - & - & - & $7.00 \pm 0.00$ & $7.00 \pm 0.00$ & $8.00 \pm 0.00$ & $7.00 \pm 0.00$ & $7.00 \pm 0.00$ & $7.00 \pm 0.58$ & 13 \\
\hline Salmonella typhimurium SL1344 & - & - & - & - & - & $7.00 \pm 0.00$ & - & - & - & - & - & - & 23 \\
\hline Staphylococcus aureus ATCC 25923 & $8.00 \pm 0.58$ & $9.00 \pm 0.00$ & $10.00 \pm 0.58$ & - & - & - & - & - & - & - & - & - & 24 \\
\hline Staphylococcusepidermidis DSMZ 20044 & - & $7.00 \pm 0.00$ & $8.00 \pm 0.00$ & - & $7.00 \pm 0.00$ & $8.00 \pm 0.00$ & - & - & - & $7.00 \pm 0.00$ & $8.00 \pm 0.00$ & $9.00 \pm 0.58$ & 25 \\
\hline
\end{tabular}




\section{IV.SONUC}

Bu çalışmada $P$. elatum, R. megapolitanum, T. fasciculata, T. tortuosa olmak üzere dört farklı karayosunu örneği kullanılmıştır ve yapılan test sonucunda en iyi antimikrobiyal etki 13 farklı suşa karşı etki gösteren T. tortuosa örneğinden alınmıştır. P. elatum türünün S. aureus suşuna karşı oluşturduğu 8, 9 ve $10 \mathrm{~mm}$ zonlar da önemli sonuçlardan biridir.

Sonuçlar üzerinde gerçekleştirilen istatistiksel analiz sonucunda test edilen örneklerin paralelleri arasındaki fark istatistiksel olarak incelenmiştir. Çalışmada kullanılan paralel çalışmaların birbirinden istatistiksel olarak farklı olmadığı görülmüştür ( $\mathrm{p}>0,05)$. Ayrıca örneklerin farklı konsantrasyonlarına ait antimikrobiyal aktiviteleri arasındaki fark da istatistiksel olarak incelenmiş ve P. elatum $(\mathrm{p}=0,4738)$, R. megapolitanum $(\mathrm{p}=0,3399)$ ve $T$. fasciculata $(\mathrm{p}=0,6304)$ için farkın önemli olmadığı görülürken; T. tortuosa' da $(\mathrm{p}=0,04692)$ konsantrasyonlarının antimikrobiyal aktiviteleri arasında istatistiksel olarak fark olduğu belirlenmiş ve yapılan Pearson korelasyon analizi sonucunda dozaj artışı ile antimikrobiyal etki arasında zayıf bir korelasyon $(\mathrm{p}=0,31889)$ olduğu sonucuna ulaşılmıştır.

Savaroglu ve arkadaşları [18] tarafından yapılan çalışmada $T$. tortuosa örneğinin 7 farklı çözücü kullanılarak hazırlanan ekstraktları 6 farklı bakteri ve 3 farklı maya üstünde antimikrobiyal aktiviteleri belirlenmiştir. Çalışmada kullanılan bakteri türleri ortak olmakla birlikte farklı olarak bu çalışmada $T$. tortuosa örneğinin etanol ekstraktı kullanılmıştır. Yapılan bu çalışmada ortak suşlar olan B. subtilis, E. faecalis, E. coli, S. typhimurium, S. aureus'a karşı alınan sonuçlar Savaroglu ve arkadaşları tarafından yapılan çalışma ile paralellik göstermekte ve farklı çözücü ekstraktı olarak sonuçları genişletmektedir.

Karpiński ve Adamczak [19] tarafından gerçekleştirilen araştırmada 12 farklı karayosunu örneğinin etanol ekstraktlarının 5 farklı mikroorganizma üzerinde antimikrobiyal aktivitelerinin belirlenmiştir. İki çalışmada ortak olan E. faecalis, E. coli, S. aureus ve K. pneumoniae suşlarına karşı alınan sonuç karşılaştırıldığında bu çalışmada kullanılmış olan P. elatum, R. megapolitanum, T. fasciculata, $T$. tortuosa örnekleri Karpiński ve Adamczak tarafından yapılan çalışmadaki 5 karayosunu örneğinde daha düşük, 3 karayosunu örneği ile eşit ve 4 karayosunu örneğinde ise daha yüksek antimikrobiyal aktivite göstermiştir.

Ertürk ve arkadaşları [20] tarafından 8 farklı karayosunu türünün etanol ekstraktlarının 10 bakteri ve 3 maya suşuna karşı antimikrobiyal aktivitelerinin belirlendiği çalışmada kullanılan 6 bakteri ve 1 maya türü bu çalışma ile ortaktır. Bu çalışmada kullanılan karayosunu örnekleri iki çalışmada kullanılan ortak olan E. coli, K. pneumoniae, L. monocytogenes, S. aureus, S. typhimurium, P. aeruginosa, C. albicans suşlarına karşı diğer çalışmadaki örneklerden daha az antimikrobiyal aktivite göstermiştir.

Olasoji ve arkadaşları [21] gerçekleştirdiği çalışmada 3 farklı karayosunu örneğinin etanol ekstraktlarının 4 bakteri ve 2 maya suşuna karşı antimikrobiyal aktivite belirlenmiştir. Çalışmada kullanılan E. coli, B. subtilis, S. aureus, P. aeruginosa, C. albicans suşları her iki çalışma içinde ortaktır. Gerçekleştirilen çalışmada kullanılan 3 farklı karayosunu örneği ortak suşlara karşı antimikrobiyal etki göstermezken bu çalışmada kullanılan karayosunu örnekleri sadece C. albicans suşuna karşı etki göstermemiş, kalan suşlara karşı etki göstermiştir.

$\mathrm{Bu}$ çalışma ile antimikrobiyal aktivitesi belirlenen karayosunu örneklerinin ortaya koyduğu etkilerin madde miktarının arttırıldıktan sonra testler tekrar edildiğinde daha yüksek antimikrobiyal aktivite göstermesi beklenilmektedir. Karayosunu örneklerinin ekstraklarında saflaştırma işlemi gerçekleştirilmediği için ekstraktların sahip oldukları etken madde miktarı bilinmemektedir. Daha sonra yapılacak bir saflaştırma işlemi sonrasında dozaj çalışmaları yapılarak görülen antimikrobiyal etkiden 
pratikte faydalanılıp faydalanılamayacağı daha net olarak ortaya konulabilecektir. Karayosunu örneklerinin sahip olduğu aktif bileşiklerin farmakokinetik etki mekanizmaları ve antibiyotik etkileşimlerinin tespit edebilmesi için daha kapsamlı araştırmalara gerek duyulmaktadır.

Not: Bu çalışmada kullanılan Plagiomnium elatum ve Rhynchostegium megapolitanum örnekleri poster bildiri olarak Mayıs 2017 tarihlerinde Konya'da düzenlenen 1. International Congress on Medicinal and Aromatic Plants 'de sunulmuş ve özet metin olarak kongre kitabında yer almıştır. Tortella fasciculata (Posterde türün önceki ismi olan Tortella bambergeri kullanılmıştır) ve Tortella tortuosa örnekleri de poster bildiri olarak 11-13 Mayıs 2017 tarihlerinde Kayseri'de düzenlenmiş olan ECOLOGY 2017'de sunulmuş ve özet metin olarak kongre kitabında yer almıştır.

\section{KAYNAKLAR}

[1] B. Dülger, A. Gönüz, "Antimicrobial Activity of Certain Plants Used in Turkhish Traditional Medicine," Asian Journal of Plant Sciences, vol.3, no.4, pp.104-107, 2004.

[2] A. E. Erdoğan, A. Everest, "Antimikrobiyal Ajan Olarak Bitki Bileşenleri," Türk Bilimsel Derlemeler Dergisi, c.6, s.2, ss.27-32, 2013.

[3] E. Faydaoğlu, M. Sürücüoğlu, "T1bbi ve aromatik bitkilerin antimikrobiyal, antioksidanaktiviteleri ve kullanım olanakları," Erzincan Üniversitesi Fen Bilimleri Enstitüsü Dergisi, c.6, s.2, ss.233-265, 2013.

[4] S. Ursavaş, R. Söyler, "Quantities and sales data of harvested mosses base on Regional Directorate of Forestry," Kastamonu Üniversitesi Orman Fakültesi Dergisi, vol.15, no.2, pp. 241-251, 2015.

[5] E. M. Altuner, B. Çetin, "Antimicrobial activity of Isothecium alopecuroides and potential effect of some climate elements on the activity of this bryophyte sample," Kastamonu Universitesi Orman Fakültesi Dergisi, vol.18, no.2, pp.126-137, 2018.

[6] S. Ursavaş, (2009) "Karayosunları ve ciğerotlarında tür ve çeşitlilik," Ankara Üniversitesi Biyoloji Anabilim Dalı, seminer kitabı, ss. 1-45.

[7] S. Ursavaş, Z. Işın, "New records of Bryum gemmiferum and Atrichum crispum for Turkey," Plant Biosystems-An International Journal Dealing with all Aspects of Plant Biology, vol. 5, no.153, pp.686-690, 2019.

[8] A. Erdağ, H. Kürschner, "Türkiye bitkileri listesi, Karayosunları: eklentiler 2016-2017," Bağbahçe Bilim Dergisi, c.5, s.2, ss.10-16, 2018.

[9] E. M. Altuner, B. Çetin, B. Çökmüş, "Tortella tortulosa (Hedw.) Limpr. Özütlerinin Antimikrobiyal Aktivitesi," Kastamonu Üniversitesi Orman Fakültesi Dergisi, c.10, s.2, ss.111-116, 2010.

[10] K. Canl1, A. Yetgin, I. Akata, E. M. Altuner, "Antimicrobial activity and chemical composition screening of Epilobium montanum root," Indian Journal of Pharmaceutical Education and Research, vol. 51, no. 3, pp. 239-243, 2017. 
[11] K. Canl1, E. M. Altuner, I. Akata, "Antimicrobial screening of Mnium stellare," Bangladesh Journal of Pharmacology, vol.10, pp. 321-325, 2015.

[12] E. M. Altuner, K. Canl1, I. Akata, "In vitro Antimicrobial Screening of Hedwigia ciliata var. leucophaea and Determination of the Ethanol Extract Composition by Gas Chromatography/Mass Spectrometry (GC/MS), "Journal of Pure and Applied Microbiology, vol. 4, no. 8, pp.2987-2998, 2014.

[13] B. Baldaş, E. M. Altuner, "The antimicrobial activity of apple cider vinegar and grape vinegar, which are used as a traditional surface disinfectant for fruits and vegetables," Communications Faculty of Sciences University of Ankara Series C Biology, vol. 27, no.1, pp.1-10, 2018.

[14] J. M. Andrews, "BSAC standardized disc susceptibility testing method (version 6), "Journal of Antimicrobial Chemotherap, vol.60, pp. 20-41. 2003.

[15] K. Canl, A. Yetgin, I. Akata, E. M. Altuner, "In vitro Antimicrobial Activity of Angelica sylvestris Roots,” International Journal of Biological Sciences, vol.1, no.1, pp.1-7, 2016.

[16] M. E. Bozyel, M. Şenturan, A. Benek, E. Bozyel Merdamert, K. Canl1, E. M. Altuner, "In Vitro Antimicrobial Activity Screening of Heliotropium europaeum Against Wide Range of Microorganisms and Multi Drug Resistant (MDR) Bacteria," European Journal of Biomedical and Pharmaceutical Sciences, vol.6, no.3, pp.113-117, 2019.

[17] Core R Team, R: A language and environment for statistical computing. R Foundation for Statistical Computing, https://www.R-project.org/ (last accession: 15.08.2019).

[18] F. Savaroğlu, S. İlhan, C. Filik, İşcen, "An evaluation of the antimicrobial activity of some Turkish mosses," Journal of Medicinal Plants Research, vol.5, no.14, pp. 3286-3292, 2011.

[19] T. M. Karpiński, A. Adamczak, "Antibacterial activity of ethanolic extracts of some moss species” Herba Polonica, vol.63, no.3, pp.11-17, 2017.

[20] Ö. Ertürk, H. Şahin, E. Ertürk, H. E. Hotaman, B. Koz, Ö. Özdemir, "The antimicrobial and antioxidant activities of extracts obtained from some moss species in Turkey," Herba Polonica, vol.61, no.4, pp.52-65, 2015.

[21] K. O. Olasoji, A. M. Makinde, B. A. Akinpelu, O. A. Igbeneghu, "Antimicrobial Activity of Selected Mosses on Obafemi Awolowo University Campus, Ile-Ife, Nigeria," Notulae Scientia Biologicae, vol.11, no.3, pp.462-466, 2019. 